\title{
Satiation of a verbal cue in problem solving in French speaking children
}

HAROLD COOK AND JEAN VACHON, DEPARTMENT OF PSYCHOLOGY, SYRACUSE UNIVERSITY, Syracuse, N. Y. 13210

Forty French speaking fourth grade children were randomly assigned to groups and repeated either a relevant cue, the word "corde" (string), or an irrelevant cue, the word "porte" (door) for $1,15,30$ or 60 repetitions prior to attempting to solve a measurement of circumference problem. The solution of the problem involved the use of a string. Latency to solution of the problem was statistically greater for the groups who continually repeated the relevant cue as compared to those who continually repeated the irrelevant cue.

Semantic satiation has in recent years attracted increased research interest. The phenomenon is generally defined as the loss in intensity of meaning of a word, following its continued repetition or prolonged exposure (Amster, 1964). In attempting to discover the range of applicability, the delineations of its parameters, and an explanation underlying the phenomenon, a wide range of tasks and variety of conditions have been subjected to study. Most of the reported investigations have used adults as Ss.

Wakin \& Braun (1966) demonstrated that a group of college students who repeated the word "string" for $30 \mathrm{sec}$ prior to attempting the Maier two-string problem took longer to solve the problem than either a control group who repeated the word "small," or a control group that had no satiation treatment.

The present investigation attempted to study parametrically the effect of satiation of a verbal cue on a subsequent problem solving task in children. It was hypothesized that the continued repetition of a relevant (to the solution of the problem) word would interfere with subsequent problem solving, as compared to the repetition of an irrelevant word. Further, that there would be a curvilinear relationship between the number of repetitions and latency to solve the problem, such that for a single repetition problem solving would be facilitated, for 15 and 30 repetitions a decrement would occur, and a reversal in the decrement would be evident at 60 repetitions.

Subjects. The Ss were 40, fourth-grade French speaking children from a public elementary school in Quebec City. The 19 boys and 21 girls had a mean age of 112.7 months and were randomly assigned to one of eight groups.

Apparatus and Materials. A standard clothesline pulley, with the grooved center of the pulley wheel painted white, a l-ft wooden ruler and a piece of clothesline 15 in. long were placed behind a screen on a small wooden table. A stop watch, pencil, and data sheets were also available.

Design. The design was a 2 by 4 complete factorial design. The factors were (a) the word repeated, either the relevant cue "corde" (string) or the irrelevant cue "porte" (door); and (b) the number of repetitions, either 1, 15, 30 or 60 . The measure of the dependent variable was the latency, time in sec, taken to solve the problem

Procedure. Each S was taken out of his class into the experimental room and run individually. The experiment consisted of two phases: (1) E engaged $S$ in friendly conversation and seated $S$ at a table supporting the apparatus and administered the instructions that follow, which were translated into French. The E was a native-born French speaking Canadian. "Hello, my name is____ What's your name? Today we will play a couple of games. It is easy, but you will have to think a little bit about it. First, we will play a word game. I will say a word, then I want you to say the word and keep on saying the word over and over again until I tell you to stop. For example, if I say the word "window," you would say: "window, window, window..." without stopping. Do you understand? I may ask you to "stop" after you have said the word only once or I may not say "stop" until you have repeated it many times. O. K.? Now say "string" ("door" for the irrelevant cue groups) until I tell you to stop."

The E said "stop" when S had repeated the word for the appropriate number of repetitions. (2) E then removed the screen and placed the puliey, the ruler, and the string in front of S. E held the pulley and said: "We will now play a different game. You will have a problem to solve. I'd like you to find a way to measure the circumference of this wheel along the white line. (E showed the pulley wheel to $S$.) You can use anything on the table. Do you understand? $\mathrm{O}$. K. Begin." $\mathrm{E}$ began timing and let $\mathrm{S}$ work until he solved the problem. If $S$ did not find the appropriate solution, $E$ asked him to stop after $3 \mathrm{~min}$. If $\mathrm{S}$ found an inappropriate solution before $3 \mathrm{~min}, \mathrm{E}$ said: "Try to find another way to solve the problem."

The $\mathbf{S}$ was credited with solving the problem if he found a way to measure the circumference. E considered the problem solved correctly if $S$ put the string around the white line of the pulley wheel and then measured the string with the ruler, regardless of the accuracy of the measurement. The above method of solution was the criterion, not the accuracy of measurement. E recorded the time taken to solution to the nearest sec. For those Ss unable to solve the problem within the 3 min maximum time limit, $180 \mathrm{sec}$ was recorded as their latency score.

Results and Discussion. A two-way analysis of variance was performed on transgenerated means of the time taken to solve the problem. Each score was transgenerated by $\sqrt{ } x+\sqrt{ } x+1$. The factors were: (1) the word, either relevant cue or irrelevant cue and (2) the number of repetitions, either 1, 15,30, or 60 . The analysis yielded statistically significant $F$ values for the repeated word $(F=6.56, \mathrm{df}=3 / 31, \mathrm{p}<.05)$.

Results of the $F$ tests demonstrated that the groups that repeated the relevant cue took significantly longer to solve the problem than the groups that repeated the irrelevant cue. Furthermore, out of the $20 \mathrm{Ss}$ in the relevant cue satiation condition, 11 failed to solve the problem in the allotted $3 \mathrm{~min}$ compared to only five out of $20 \mathrm{Ss}$ in the irrelevant cue satiation condition. This result supports the first hypothesis that satiation of a relevant cue interfered with subsequent problem solving as compared to irrelevant cue satiation.

Trend analysis (Winer, pp. 70-77) revealed only one statistically significant trend, a cubic trend for the irrelevant cue across number of repetitions. The trend analysis results fail to support the second hypothesis regarding the curvilinear relationship between number of repetitions and latency to solve the problem. The cubic trend generated by the repetition of the irrelevant cue cannot be presently explained.

Means for the time taken to solve the problem were for the relevant cue condition, in sec, 129.60 for one repetition, 107.10 for $15,132.40$ for 30 , and 165.00 for 60 repetitions. In the irrelevant cue condition the means for $1,15,30$, and 60 repetitions were $133.40,37.00,134.40$, and $68.60 \mathrm{sec}$, respectively.

A second two-way analysis of variance was performed on the mean latency scores. The factors were: (1) sex of S, and (2) the satiated word, either a relevant cue or irrelevant cue. This analysis yielded only one significant $F$ for the intersection of satiated word and $\operatorname{sex}(F=4.23, \mathrm{df}=1 / 36, \mathrm{p}<.05)$. This suggests that there is greater interference (longer time taken to solve the problem) for girls than for boys when the satiated word is a relevant cue. The opposite occurs when the satiated word is an irrelevant cue, the problem is solved more quickly by girls than boys.

The results of the study appear to be in accord with those obtained by Wakin \& Braun (1966), and extend the generality of their findings to children. The findings suggest that the satiation of a relevant cue (mediator) had significant inhibitory effect on the solution of a measurement problem.

The interesting, statistically significant interaction of sex by satiated word suggests the need for additional work dealing with sex differences and satiability. Further exploration of the satiation phenomenon in children may also lead to an increased understanding of the acquisition of the meaning of words, and the relationship of language to problem solving.

\section{REFERENCES}

AMSTER, H. Semantic satiation and generation: learning? adaptation? Psychol. Bull., 1964, 62, 273-286.

WAKIN, A. H., \& BRAUN, J. P. Semantic satiation and problem solving. Psychon. Sci, 1966, 5, 469.

WINER, B. J. Statistical principles in experimental design. New York: McGraw-Hill, 1960. 